\title{
L'IMPORTANCE DE LA SEPTANTE DANS UNE NOUVELLE ÈRE DE LA CRITIQUE TEXTUELLE *
}

The importance of the Septuagint in a new era of textual criticism

Bénédicte Lemmelijn **

RÉSUMÉ: L'article traite de deux questions étroitement liées: la compréhension changée de la critique textuelle de la Bible aujourd'hui, et l'étude ainsi que l'interprétation de la Septante (LXX) dans le cadre de cette situation changée. Pendant longtemps le Texte Massorétique (TM) a été considéré comme le témoin principal du texte hébraïque " originel ", mais l'attention dédiée à la LXX, la révalorisation des versions anciennes et, surtout, la découverte des manuscrits de la mer Morte, qui constituent des témoins hébraïques bien antérieurs au TM et confirmant quelques fois la LXX, posent la question s'il faut parler d'un texte originel ou d'une convergence de meilleurs témoins. Quant à la LXX, actuellement, on prête attention aux variantes hébraïques sous-jacentes et à l'activité « rédactionnelle » du traducteur. La LXX n'est plus simplement la « ancilla hebraicae veritatis » mais un précieux témoin autonome au sein d'une multiplicité pluriforme de preuves textuelles.

MOTS CLÉS: Septante. Critique textuelle. Variantes. Texte Massorétique. Texte original.

ABSTRACT: The article presents two interconnected issues: the modified comprehension of Textual Criticism of the Hebrew Bible at the present moment and the study and interpretation of the Septuagint (LXX) in this new context. For a long

\footnotetext{
* Cet article est basé sur une contribution anglaise, plus détaillée, qui paraîtra comme: B. LEMMELIJN, Textual Criticism, dans A. SALVESEN \& M. LAW (eds.), Oxford Handbook of the Septuagint, Oxford: Oxford University Press, 2020, sous presse. Pour une version plus simple, qui ne présente que la première partie, voir B. LEMMELIJN, A la recherche du texte de la Bible Hébraique, dans L’Homme Nouveau Hors-Série, nr. 34 (2019) 21-28. Nous remercions le Drs. Augustin Lwamba pour son aide au niveau du français.

** Katholieke Universiteit Leuven, Leuven, Belgium.
} 
time, the Masoretic Text (MT) has been considered as the main textual witness of the "original" Hebrew text. However, the renewed attention for the LXX, the revalorization of the ancient versions and especially the discovery of the Dead Sea Scrolls, Hebrew witnesses far older than the MT, eventually confirming variant readings in the LXX, raise the question whether we should speak about the convergence of the main and the best textual witnesses rather than about an "original text". Concerning the LXX, actually, attention is given to the subjacent Hebrew variants and the "redactional" activity of the translator. LXX should no longer be seen as the "ancilla hebraicae veritatis", but rather as an autonomous version and a highly valuable witness within a multiplicity of pluriform textual evidences.

KEYWORDS: Septuagint. Textual Criticism. Variants. Masoretic Text. Original text.

\section{Introduction}

$I^{1}$ l fut un temps où il était assez facile de définir la " critique textuelle » ainsi que de déterminer ses objectifs et ses méthodes. L'idée " moderne » de la critique rationnelle et unilatérale était confiante de reconstruire sans ambiguïé le développement du texte biblique jusqu'à son unique point d'origine. Cependant, l'augmentation de l'évidence des faits et le développement de la compréhension nous ont amenés aujourd'hui à une situation beaucoup plus compliquée. Dans ce contexte, la place de la Septante - la première traduction grecque de l'Ancien Testament - dans le processus de la critique textuelle de la Bible Hébraïque a également changée de manière indéniable.

Dans les paragraphes qui suivent, je décris d'abord comment les « faits » textuels ont changé notre compréhension du développement du matériel textuel biblique et comment ils ont aussi changé notre vision ainsi que notre perception de la critique textuelle elle-même. Ensuite, je focalise sur le rôle qu'a joué la Septante dans ce panorama changeant. Enfin, je conclurai en démontrant cette signification altérée de la Septante sur base d'un exemple textuel concret.

\section{La critique textuelle dans la situation textuelle actuelle}

\subsection{Toutes les bonnes choses viennent par trois... La situation textuelle et l'interprétation savante avant les découvertes de la mer Morte}

Pendant longtemps, le «Texte Massorétique » (TM) a servi de principal témoin pour le texte hébreu de l'Ancien Testament (TOV, 2012a, p. 155160; LEMMELIJN, 2009, p. 1-28; DEBEL, 2012, p. 205-213). Ce texte a été 
conservé principalement dans le codex de Léningrad - appelé parfois le codex de Saint-Petersbourg ou encore le codex Petropolitanus - du XIe siècle après J.-C., et également dans le codex d'Alep du Xe siècle après J.-C., qui tout en étant incomplet, est donc légèrement plus ancien. À côté du TM, un autre texte hébreu était disponible dans le Pentateuque Samaritain (PentSam) (manuscrits du IXe au XIIIe siècle), mais comme le suggère le nom lui-même, il ne proposait que le texte du Pentateuque. Cela implique une absence totale d'anciens témoins textuels hébreux. En effet, tous ces manuscrits ont jusqu'à douze siècles de moins que la datation généralement acceptée des dernières compositions bibliques datant du IIIe siècle avant J.-C.

Par conséquent, afin de pénétrer davantage la situation textuelle de ce moment-là, les chercheurs ont eu recours à l'étude des traductions anciennes (TOV, 2012a, p. 17-19). À cet égard, un troisième témoin textuel utilisé pour étudier le texte biblique était la Septante (LXX). Cette traduction grecque ancienne n'a pas seulement offert un texte complet (voir les principaux codices Vaticanus, Sinaiticus et Alexandrinus, IVe-Ve siècle après J.-C.), mais aussi un texte beaucoup plus ancien que les textes hébreux. En effet, étant daté à l'origine au IIIe siècle avant J.-C., il était vieux de plus de mille ans que les témoins hébreux (le TM et le PentSam). Les traductions " issues » de la LXX, telles que l'Éthiopien, l'Arménien, le Copte et la Vetus Latina, ont également attiré l'attention des chercheurs. À côté de l'intérêt principal pour la Septante, les autres versions telles que la Vulgate, la Peshitta ainsi que les Targumim ont commencé à jouer un rôle dans l'étude des textes bibliques. Cependant, après un certain temps, les chercheurs se sont vite aperçus que toutes ces versions étaient plus d'un grand intérêt à l'exégèse biblique plutôt qu'à la critique textuelle.

Compte tenu de cette situation textuelle, les chercheurs se sont basés sur les principaux témoins textuels, à savoir le TM, le PentSam et la LXX. Ils ont regroupé les autres témoins textuels existants et leurs traductions (secondaires) dans l'un des trois principaux «types de textes ", "familles textuelles ", "recensions textuelles » de l'un des trois " groupes » (TOV, 2012a, p. 155-159; cf. aussi CROSS 1995, 1998; ALBRIGHT, 1955; SKEHAN, 1965; TALMON, 1970; MILIK, 1957; DAVILA, 1993). Partant de cette triple division, les spécialistes de la critique textuelle ont tenté d'établir «la variante originale», aussi bien sur le niveau des manuscrits/textes en tant que tels (évaluation externe), que sur le niveau des variantes individuelles au sein des différents témoins textuels (évaluation interne) (LEMMELIJN, 2009, p. 15-18). De cette façon, ils ont tenté de reconstruire le développement textuel de la Bible hébraïque.

Dans cette recherche, deux positions opposées ont été avancées (TOV, 2012a, p. 156). D'une part, Paul de Lagarde affirmait qu'il y avait eu en hébreu un seul texte original qui aurait servi de modèle à l'aube de la transmission textuelle, et parallèlement à cela, il existait aussi un seul texte 
grec qui aurait été la proto-Septante, comparable à ce qui est aujourd'hui appelé le «Old Greek»(OG). Ces deux textes auraient été précédés d'un « ancêtre » commun, qui aurait été $l^{\prime}$ « Urtext » de tous les textes bibliques (DE LAGARDE, 1863). D'autre part, Paul Kahle ne croyait pas à l'existence de cet «Urtext » unifié. Il affirmait qu'une multiplicité de textes était à la base autant du texte hébreu que du texte grec (KAHLE, 1915). Pour le texte en hébreu, différents "Vulgärtexte » auraient circulé pour fournir au peuple du matériel textuel, tandis que pour les textes grecs, ils auraient été traduits selon le besoin des communautés, niant ainsi l'existence d'une seule proto-Septante.

Toutefois, malgré les points de vue divergents de Kahle et de Lagarde qui soutiennent soit l'existence d'une multiplicité de textes, soit la présence d'un seul et unique texte original au début de la tradition textuelle, l'hypothèse de la critique textuelle à la recherche de la « variante originale » consistait dans l'acceptation d'un «Urtext » ou du moins du premier stade de la rédaction du texte que l'on pourrait atteindre en reconstruisant ce texte original (TOV, 2012a, p. 161-169; TOV, 2012b; LEMMELIJN, 2009, p. 1-2,25-27; LEMMELIJN, 1997, p. 69-80; DEBEL, 2011b). Le " meilleur » texte (ou la variante " préférable ») serait donc celui/celle qui est le plus proche de ce texte « original ».

\subsection{De trois à plusieurs. La preuve textuelle des manuscrits de la mer Morte change le panorama textuel}

Même de nos jours, la valeur du TM, du PentSam et de la LXX n'est pas mise en doute. Ces témoins textuels sont encore considérés dans une certaine mesure comme les principales sources de nos recherches sur le développement des textes bibliques et leur transmission. Cependant, les découvertes faites dans la région de la mer Morte entre 1947 et 1956 ont changé le panorama des chercheurs sur ces textes bibliques (TOV, 2012a, p. 157-160; WHITE CRAWFORD, 2012a, 2012b).

En effet, après la découverte des manuscrits de la mer Morte, il est devenu de plus en plus clair que la division tripartite mentionnée ci-dessus ne reflétait nullement la réalité des faits égard à l'existence d'une variété beaucoup plus large de multiples « textes » individuels (TOV, 1982), ayant leurs propres particularités qui ne sont pas nécessairement partagées par d'autres textes, et qui ne relèvent aucun « modèle » spécifique en fonction duquel ils pourraient être regroupés dans l'une des trois " recensions » mentionnées plus haut.

Cette variété de matériaux textuels aux multiples facettes a conduit les chercheurs à la conclusion que le matériel textuel de la période du Second Temple fait preuve aussi bien de pluralité que de pluriformité (DEBEL, 2010 et 2011a). D'où l'intérêt à étudier les textes pour eux-mêmes. Partant de la découverte des manuscrits de la mer Morte, il n'est plus possible 
de soutenir que le TM, le PentSam et la LXX constituent les seules principales recensions auxquelles tout le reste des textes devrait être attribué. Les manuscrits de la mer Morte reflètent plutôt une multiplicité de textes différents et particuliers (TOV, 2012a, p. 158-159). En effet, certains reflètent le TM, le PentSam ou la LXX, mais il existe aussi un nombre assez important de "non-aligned texts » qui témoignent de l'existence d'autres textes particuliers (TOV, 2012a, p. 107-110; 159-160).

Cette variété des témoins textuels renforce aussi les doutes sur ce qu'on appelle l'Urtext. Car, dans cette configuration, postuler l'existence en amont d'une multiplicité de textes s'est montré une meilleure alternative.

\subsection{La tâche et le but de la critique textuelle changent}

Cette constatation avait, bien évidemment, aussi des conséquences en ce qui concerne la tâche et le but de la critique textuelle. Devraient-ils être non pas la reconstruction de l'original, mais plutôt la relation entre tous les témoins textuels existants actuels, même ceux qui sont partiellement et accidentellement conservés (TOV, 2012a, p. 159)? A cet égard, la critique textuelle viserait, d'une part, à collecter et décrire toutes les différences (appelées variantes) observées dans le matériel textuel existant d'une certaine péricope, et à définir ainsi leur pertinence par rapport au texte (par exemple, distinguer les " grammatical variants » des « text-relevant variants » (LEMMELIJN, 2009, p. 13-20; 150-151). D'autre part, elle consisterait en fin de compte à évaluer ces variantes les unes par rapport aux autres afin de déterminer en présence des deux variantes, celle qui provient de l'autre et, ce faisant, de choisir celle qui serait " préférable ». Dans les cas où aucune "préférabilité » ne peut être énoncée, on parle de variantes « synonymes » (LEMMELIJN, 2009, p. 20-22).

Cependant, dans ce processus de définition de la " préférabilité », la perspective de reconstruire ou de trouver la lecture « originale » devrait céder la place à un cadre " relatif » dans lequel les variantes existantes peuvent être évaluées les unes par rapport aux autres. En même temps, il ne faut pas rêver d'un "Urtext », puisque ce dernier peut être laissé au domaine de la "préhistoire » textuelle (LEMMELIJN, 2009, p. 22-27; LEMMELIJN, 1997). La tendance actuelle, consistant à se focaliser sur les témoins textuels disponibles et réels dans toute leur multiplicité et leur pluriformité, invite la critique textuelle à étudier d'abord la relation entre les textes existants et à comprendre finalement la fluidité d'une tradition dynamique qui était à leur origine (ULRICH 1999; TOV, 2009).

\subsection{Changer d'objectif, c'est changer de voie}

Il va de soi que la prise en compte de cette variété des textes bibliques suite aux découvertes de la mer Morte, apporte de changement non seu- 
lement dans le but ou la tâche de la critique textuelle, mais aussi dans sa méthodologie. Cela va jusqu'à altérer en particulier l'appréhension de la relation entre la critique textuelle et la critique littéraire (LEMMELIJN, 2012, p. 203-207).

En effet, à côté de la multiplicité et de la pluriformité du matériel textuel, l'étude des manuscrits de la mer Morte a généré l'idée que les différences textuelles auxquelles les chercheurs étaient confrontés n'étaient pas nécessairement des changements (mineurs) qui se seraient glissés lors du processus de transmission, mais que ces différences sont aussi parfois le fruit d'une sérieuse activité rédactionnelle, interprétative et même exégétique. D'où la prise de conscience du fait que les «frontières » entre le domaine de la « critique textuelle » et celui de la " critique littéraire » n'étaient pas du tout aussi nettes qu'on le croyait auparavant. En effet, jusque-là, les chercheurs avaient l'habitude de considérer la « critique textuelle » comme l'étude du processus de transmission du texte. Cette étape de transmission du texte n'aurait eu lieu qu'après la phase de production/composition littéraire qui correspond au stade de finalisation du texte qui est du ressort de la « critique littéraire ».

De nos jours, ce point de vue est remis en question par la réalité des textes mêmes (TREBOLLE BARRERA, 1998, p. 370, 390; idem, 2005, p. 413). En effet, une distinction claire entre ces deux processus de composition littéraire et de transmission textuelle ne peut tout simplement pas être faite de manière satisfaisante (LEMMELIJN, 2009, p. 3-7 et passim; idem, 2012, p. 204-205). Car, premièrement, ces deux étapes se chevauchent en fait dans la création des textes. Il est même probable que la transmission textuelle de certains textes bibliques était déjà en cours avant l'achèvement de la composition littéraire en question, si jamais un tel achèvement était atteint consciemment ou intentionnellement. Deuxièmement, il est clair que lorsque la critique textuelle et littéraire " coopèrent » dans l'étude du texte, les irrégularités et les problèmes littéraires sont souvent découverts précisément dans les cas où des variantes textuelles sont observées (LEMMELIJN, 2001, p. 429-439; LEMMELIJN, 2009, p. 197-207; TREBOLLE BARRERA, 2008, p. 437-463).

De plus, ce fait ne soulève pas seulement des questions concernant la distinction susmentionnée entre les deux domaines de la critique textuelle et littéraire en tant que telle. Il conteste aussi sérieusement l'affirmation plutôt spéculative dans l'ensemble concernant une prétendue différence de méthode entre les auteurs ou les rédacteurs d'une part, et les scribes ou les copistes d'autre part (SCHENKER, 2012, p. 275-298).

Dans ce contexte, il devient clair que la recherche d'un "Urtext ", autrefois considérée comme le but ultime de la critique textuelle, n'est plus valable. Aussi l'acceptation implicite d'un tel principe par les chercheurs qui, dans le cadre de leur étude littéraire, structurelle, diachronique ou 
synchronique d'une péricope spécifique, se contentent de traiter le TM comme s'il s'agissait du texte original, passe-t-elle pour encore moins critique. En effet, parler du canon de l'Ancien Testament et a fortiori parler d'un " texte standard » normatif de l'Ancien Testament - parfois pour des raisons confessionnelles - ne peut plus être maintenu dans le cadre actuel d'une prise croissante de conscience sur l'existence de nombreuses et multiples preuves textuelles évidentes.

\subsection{Implications méthodologiques}

Fort de ce qui précède, il convient de tirer un certain nombre de conclusions méthodologiques qui, néanmoins, ne sont souvent pas prises dans la pratique (LEMMELIJN, 2012, p. 205-207; cf. aussi BROOKE, 2005, p. 26-42):

a. Puisque $1^{\prime}$ «Urtext » n'existe pas, nous ne pouvons pas non plus parler de lecture " originale » même dans le cas des variantes individuelles. Au contraire et dans un cadre "relatif » des témoins textuels (conservés accidentellement), nous ne pouvons parler que de la variante «plus originale » (LEMMELIJN, 2009, p. 22-27; LEMMELIJN, 1997, p. 69-80).

b. Par extension, cela signifie que la volonté de reconstruire des textes " éclectiques », comme s'ils pouvaient représenter un texte s'approchant de l'« original », n'est plus souhaitable en tant que tel. Il faut être très prudent et prendre sérieusement conscience des problèmes liés à une telle reconstruction. A fortiori, ce serait aussi déconseillé dans le but d'établir un texte critique comme point de départ d'une étude littéraire des péricopes spécifiques (LEMMELIJN, 2009, p. 215). D'un point de vue théorique, on pourrait en effet affirmer que, si l'on pousse à l'extrême une telle évaluation critique des variantes d'un texte spécifique, on aboutirait nécessairement à un texte "nouveau » et éclectique contenant toutes les variantes "préférables » des différents témoins textuels. Et, on serait tenté de dire qu'une telle reconstruction critique sur la base de principes scientifiques pourrait offrir un réel avantage (HENDEL, 2000a, p. 197-217; HENDEL, 2000b, p. 8-11, esp. 11; HENDEL, 2007, p. 97-98; TREBOLLE BARRERA, 1998, p. 387). Néanmoins, on s'appuierait seulement sur un texte qui n'existe pas réellement, un texte basé uniquement sur une reconstitution hypothétique d'un certain nombre de manuscrits survivant fortuitement. Qui plus est, il s'agirait d'un texte " reconstruit » à partir d'une évaluation des variantes qui n'aurait pas été exempte d'un certain degré de subjectivité. Par conséquent, la seule alternative valable semble celle d'opter pour un et unique témoignage textuel qui, quoiqu'imparfait, est bien défini et au moins existant objectivement. Bien sûr, ce faisant, on sera néanmoins obligé de prendre pour point de départ, tout le matériel textuel disponible et de garder à l'esprit les observations textuelles associées à ce matériel. 
c. Si des variantes du texte critique, et en particulier les variantes « significatives » (« text-relevant », LEMMELIJN, 2009, p. 150-151), sont repérées dans des lieux d'importance littéraire et théologique, ces différentes lectures dans des manuscrits distincts ne peuvent plus simplement être classées comme erreurs et déviations de leur " original », ou «Vorlage ». Au contraire, elles constituent des témoins précieux d'une tradition textuelle spécifique.

d. Enfin, et en conséquence du point précédent, si les différentes variantes textuelles reflètent en fait des préoccupations littéraires et/ou théologiques dans les manuscrits textuels existants (et pas seulement des erreurs), alors la différence ambiguë entre la méthode des auteurs/ rédacteurs d'une part, et celle des scribes/copistes, et par extension même celle des traducteurs d'autre part (DEBEL, 2010), n'est plus facile à discerner ou à définir, si, tout court, elle existe encore (LEMMELIJN, 2012, p. 203-222) . Après tout, si plusieurs communautés utilisaient chacune ses propres textes religieux spécifiques et concrets et les transmettaient de manière créative en les recontextualisant (cf. les idées sur "stability » et « adaptability », p. e. dans SANDERS, 1984, p. 22 ; et 1991, p. 209; ou ULRICH, 1994a, p. 84 ; et 1997, p. 335-336), quelle serait alors la différence entre la "Fortschreibung » ou la rédaction interprétative des auteurs et des rédacteurs dans les étapes « littéraires » précédentes d'une part, et d'autre part les adaptations, les réinterprétations, les changements mineurs ou majeurs des scribes et des copistes dans les étapes de «transmission » qui produisent des textes concrets pour les besoins concrets et partant de l'(auto)-compréhension de leurs communautés respectives (ULRICH, 1994b, p. 90; et 2000, p. 129-130)?

\subsection{Encore plus de questions....}

Pour conclure, et conformément à l'opinion plus récente sur la relation entre la critique textuelle et la critique littéraire, ne devrions-nous pas accepter la possibilité que l'œuvre des scribes consistait aussi en la mise à jour, la recontextualisation et l'évolution du texte - bref, le développement littéraire factuel - au travers de leur propre activité rédactionnelle? Si cette hypothèse est acceptée, on peut dès lors estimer que la démarcation stricte entre le domaine de la critique textuelle et celui de la critique littéraire, mutatis mutandis entre la création et la production du texte, ou en d'autres termes, entre le travail des copistes/scribes et celui des auteurs/rédacteurs, ne peut plus être maintenue.

Pourtant, tout cela introduit également une autre question. Si la rédaction, qui développe le progrès de la réflexion théologique, et la « Fortschreibung » faisaient partie intégrante du processus de la copie et de la transmission, l'idée de rechercher la variante " préférable » dans l'évaluation des variantes critiques d'un texte devrait-elle aussi être modifiée? En effet, d'une manière 
générale et comme indiqué plus haut, la variante préférable est considérée comme celle qui est la plus originale même dans un cadre " relatif » (voir ci-dessus) ou bien celle qui explique le développement des autres variantes. Or, si l'on affirme que la (re-)production du texte contient aussi une réflexion et une évolution au niveau du contenu théologique, la variante " préférable » peut-elle encore être " plus originale »? Cela ne créerait-il pas un paradoxe entre la lecture " préférable » dans la perspective de la critique textuelle et la variante « préférable » du point de vue littéraire? En d'autres termes, la variante "préférable » pourrait-elle ne pas être « la plus développée », d'un point de vue littéraire et théologique?

Tous ces aspects - et les conséquences qu'ils peuvent entraîner - ne prétendent nullement être une thèse avérée, ni même une hypothèse solide. Même les questions soulevées restent ouvertes (LEMMELIJN, 2012, p. 221-22). Cependant, elles ont le mérite d'être une invitation humble mais honnête adressée aux chercheurs pour qu'ils réfléchissent à des affirmations qui - trop souvent - sont tenues pour acquises. En tant que tel, il s'agit d'un appel à la (ré-) considération sérieuse des données que nous trouvons dans la multiplicité et la pluriformité des témoins textuels bibliques existants. Après tout, les données textuelles constituent le seul point de départ sûr, et par conséquent ils sont bien supérieur à une simple acceptation des (re-) constructions savantes proposées tant au niveau littéraire, rédactionnel ou textuel.

\section{2 Étudier et interpréter la Septante dans le cadre de la critique textuelle actuelle}

Lorsque nous essayons alors de saisir le sens et le rôle que la Septante a joué dans ce panorama textuel changeant et sa configuration multiforme, un changement s'observe immédiatement et tout à fait parallèlement à la compréhension du développement du texte biblique en général.

\subsection{Au fil des siècles : admirateurs et critiques....}

D'une manière générale, nous observons qu'au cours des derniers siècles, la Septante, vue comme un témoin textuel (et donc pas en ce qui concerne $l^{\prime}$ « autorité » scripturaire ou la « canonicité »), a toujours eu des admirateurs et des critiques (TOV, 1997, p. 33-34). D'une part, les uns la déprécient en attribuant pratiquement toutes ses variantes à la maladresse ou à la liberté exagérée prise par les traducteurs dans le processus de traduction (paraphrase, périphrase), négligeant ou rejetant l'idée que la Vorlage de la LXX aurait pu également s'écarter du TM (cf. par exemple l'enquête dans KÖNIG, 1893, p. 116). D'autre part, la tendance inverse 
est également observable. Jugeant le TM plutôt négativement, certains chercheurs se sont largement appuyés sur la LXX et l'ont estimé de très grande valeur (cf. par exemple, l'enquête de LEBRAM, 1975, p. 21-63, ainsi que la « citation » de F. Hitzig, dans KNEUCKER, 1880, p. 19, n.1). Bien sûr, et heureusement, un nombre de chercheurs ont pris la voie équilibrée entre les deux tendances, en prêtant attention aux variantes hébraïques sous-jacentes ainsi qu'à l'activité du traducteur.

\subsection{Septuaginta ancilla Hebraicae Veritatis : la LXX dans le modèle textuel tripartite}

Comme indiqué ci-dessus, la Septante a joué un rôle important dans la conceptualisation de l'élaboration textuelle d'un modèle textuel tripartite, axé sur trois « recensions », à savoir le TM, le PentSam et la LXX. Cependant, dans ce modèle, l'idée principale était d'atteindre le texte original. Les témoins textuels hébreux étant beaucoup plus récents que la Septante, le recours à cette dernière a été effectué dans le but de récupérer une forme du texte hébreu qui serait plus proche de ses origines. Par conséquent, la Septante n'était pas étudiée pour ce qu'elle était réellement et ce qu'elle représentait ${ }^{1}$ : elle a plutôt " servi » à l'étude du texte hébreu. Cette manière de procéder ne fait qu'accorder la prépondérance au texte hébreu. Dans ce contexte, la LXX était uniquement considérée comme une " simple " traduction, consultée principalement en raison de son âge. Par conséquent, l'étude de la Septante s'effectuait dans le cadre de l'étude du texte-critique de la péricope hébraïque qu'il fallait interpréter. En ce sens, les différentes variantes de la LXX étaient souvent utilisées, soit pour " corriger » le texte hébreu, dans les cas où celui-ci était difficile à interpréter, soit pour " adapter » le texte hébreu à un contexte littéraire et à une hypothèse exégétique.

Par ailleurs, si les variantes de la LXX n'étaient pas « utiles » dans ce sens, la plupart des variantes mineures et des expansions majeures étaient carrément attribuées aux traducteurs, sans enquête sérieuse sur la technique de traduction utilisée dans les différents livres de la Septante. C'est ici que nous atteignons un des aspects majeurs de la pertinence de la Septante dans le contexte de la critique textuelle de la Bible hébraïque. En effet, la Septante révèle un nombre assez élevé des variantes significatives. Cependant, pour être en mesure de comprendre le développement textuel du texte hébreu, comme prévu dans le plan décrit ci-dessus, et en particulier en rapport avec l'évaluation critique des textes des variantes, il reste à déterminer si

\footnotetext{
${ }^{1}$ La Septante contient d'ailleurs, à coté des livres de la Bible Hébraique traduits en Grec, également quelques livres en plus, qui, originellement, ont été écrit en Grec. Il s'agit des suppléments du livre d'Esther, des livres de Judith, Tobit, 1-4 Maccabées, le psaume supplémentaire 151, les livres des Odes de Salomon, de la Sagesse de Salomon, du Siracide, des Psaumes de Salomon, de la lettre de Jérémie, de Susanna, et enfin, de Bel et le dragon.
} 
les variantes grecques proviennent d'une Vorlage hébraïque différente ou si elles sont le résultat de l'activité du traducteur (LEMMELIJN, 2009, p. 18-20; 96-125). Il est très important d'étudier la technique de traduction utilisée dans chaque livre spécifique, séparément ainsi qu'en comparaison à d'autres, afin de pouvoir atteindre une caractérisation saine et précise de la technique de traduction de la LXX. Ce n'est que dans le contexte de cette caractérisation que l'on peut dire, avec plus ou moins de probabilité, si des variantes spécifiques sont tributaires du traducteur et découlent de son activité, ou si elles ne correspondent pas du tout à son style général et proviendraient, le cas échéant, d'une Vorlage différente.

Partant de la prise de conscience croissante de la nécessité d'une caractérisation plus précise de la technique de traduction, la Septante a progressivement commencé à être étudiée aussi pour elle-même. C'est cependant dans le sillage des découvertes de la mer Morte qui ont changé la perspective du texte critique à la fois dans son objectif et sa méthode comme décrits ci-dessus, que l'appréhension de la valeur intrinsèque de la Septante a aussi radicalement changé.

\subsection{De la 'suggestion' de la Septante au 'fait' de Qumran: la réinterprétation des variantes de LXX après la publication des manuscrits de la mer Morte}

Depuis que les découvertes dans le désert de Judée ont changé la vision des chercheurs au sujet du développement textuel de la Bible hébraïque, et surtout transformé la division tripartite simpliste des preuves manuscrites en faisant prendre de plus en plus conscience de la grande diversité et de la pluralité des textes individuels, le but et les méthodes de la critique textuelle ont également changé. En conséquence, la Septante est devenue un témoin textuel à part entière. L'enjeu, en ce sens, n'est plus celui de corriger, ni de faire des conjectures, ni d'adapter le texte de la LXX au texte hébreu, mais d'évaluer le texte de la LXX comme un témoin précieux au sein de cette grande multiplicité des preuves textuelles.

En effet, l'étude de la Septante a reçu beaucoup d'attention ces dernières décennies. D'une part, la LXX est étudiée comme un texte grec à part entière. Sa langue, ses caractéristiques stylistiques, sa théologie et son interprétation individuelle du texte biblique sont analysées. D'autre part, une attention particulière est accordée à l'étude de son caractère de traduction, et donc dans le développement de plusieurs méthodologies pour étudier le texte quantitativement et qualitativement, à la fois en termes de la grammaire et des aspects liés au contenu ( content and context related criteriology, cf. AUSLOOS ET LEMMELIJN 2010). Les résultats obtenus constituent une contribution significative à l'évaluation critique du texte des variantes de la LXX dans le contexte de la critique de la Bible hébraïque. 
Dans le sillage d'affinement de cette méthode d'étude et d'interprétation, les variantes dans la Septante ne sont donc plus considérées comme provenant toutes du traducteur, et elles sont désormais sujettes à une critique préalable. La possibilité d'existence d'une Vorlage différente est dorénavant admise comme une explication alternative légitime. Et à cet égard, un autre aspect d'importance majeure est à signaler. En fait, la Septante comportait déjà des caractéristiques qui indiquaient effectivement la diversité textuelle. Plusieurs différences dans son texte ont été d'une importance considérable. Néanmoins, il semble que le monde académique n'a réalisé l'importance des différences trouvées dans la LXX que lorsqu'il a été confronté aux données des manuscrits de la mer Morte où beaucoup de variantes principales de la LXX ont été confirmées dans une version hébraïque (TOV, 1997, p. 34-35; DEBEL ET LEMMELIJN, 2011, p. 187).

Alors qu'avant ces observations, toutes les différences étaient simplement attribuées à l'activité « libre » des traducteurs de la LXX, les textes hébraïques trouvés à Qumran ont démontré que les mêmes variantes existaient en hébreu, tout à fait simultanément avec l'origine et le développement de la LXX. Cette correspondance entre les témoins de l'ancien texte hébreu et la traduction grecque de la Septante a également augmenté l'estime de la Septante. De plus, les recherches ultérieures ont conduit à une prise croissante de conscience que la Septante a fourni des leçons à la fois dans la critique textuelle et dans la critique littéraire. En effet, les variantes grecques ne diffèrent pas seulement en tant que texte physique mais elles révèlent également des phases distinctes dans le développement littéraire du texte. Cette observation confirme même ce qui a été dit plus haut, à savoir que la démarcation entre la critique textuelle et littéraire devient moins définie à la suite de cette nouvelle façon de comprendre et d'interpréter les faits textuels.

\section{Conclusion}

Afin de rendre ce qui précède un peu plus concret, le prochain et dernier paragraphe se concentrera sur une illustration exceptionnelle dans laquelle il sera démontré comment le texte différent de la Septante, autrefois interprété sans critique comme étant tributaire de la « liberté » du traducteur, a été confirmé dans les textes hébreux trouvés à Qumran, et comment il est maintenant interprété comme reflétant une Vorlage différente révélant des phases distinctes dans le développement littéraire du texte biblique. L'exemple «par excellence » se trouve dans les différentes versions du livre de Jérémie (pour une enquête, cf. TOV, 2012a, p. 286-294; voir entre autres, BOGAERT, 1981, 1994, 2003; FISCHER, 2008; STIPP, 1994, 1997, 
2008; STULMAN, 1984, 1985). Pendant longtemps, les principales différences dans le livre de Jérémie (LXX), qui est considérablement plus court et dont l'ordre des chapitres diffère de celui du TM, étaient attribuées à un traducteur qui aurait été très libre dans la manipulation de sa Vorlage, qui ressemblerait au TM.

Cependant, quelques petits fragments de Qumran, aujourd'hui appelés $4 \mathrm{QJer}^{\mathrm{b}}$ et $4 \mathrm{QJer}{ }^{\mathrm{d}}$, offrent un texte hébreu qui diffère sérieusement du TM et qui ressemble à la Septante dans les versets et passages respectifs. En conséquence, la question de savoir si le traducteur a introduit des modifications par rapport au TM ou s'il a utilisé une Vorlage différente, semble résolue. La LXX aurait probablement été traduite à partir d'un texte hébreu qui ressemblait à $4 \mathrm{QJer}^{\mathrm{b}}$ et $4 \mathrm{QJer}{ }^{\mathrm{d}}$, et qui était probablement aussi la base du remaniement qui a finalement abouti au TM de Jérémie. De cette façon, la LXX témoigne très concrètement d'une réalité textuelle diverse, dans laquelle différentes éditions du même livre ont circulé et ont été constamment retravaillées. Ceci est d'ailleurs confirmé par le fait que, même si la LXX d'une part et $4 \mathrm{QJer}^{\mathrm{b}}$ et $4 \mathrm{QJer}^{\mathrm{d}}$ d'autre part, se ressemblent, ils révèlent simultanément aussi des différences mineures, spécialement avec 4 QJer ${ }^{\mathrm{d}}$.

Néanmoins, la question consiste à savoir comment cela s'est produit, mais aussi quel texte grec nous utilisons (DEBEL, 2012, p. 230-231). Il est possible que le traducteur n'a pas suivi sa Vorlage à la lettre, ou bien qu'il a travaillé son texte sur base d'une Vorlage légèrement différente à 4QJerd. Ou peut-être encore que le vrai «vieux grec » («Old Greek») fut un peu différent du grec ancien reconstruit dans l'édition de Göttingen par Ziegler. En tout cas, discuter de ces questions n'est plus simplement un problème de définir quel texte est « original » ou même " plus original » en termes de la critique textuelle stricto sensu. Le développement textuel, éclairé par le point de vue de la critique textuelle et attesté dans le matériel textuel existant qui révèle une tradition textuelle diversifiée et pluriforme, conduit le chercheur dans le domaine du développement littéraire du texte. De cette façon, en effet, les critiques textuelles et littéraires se chevauchent et interagissent, conduisant ainsi à une approche interdisciplinaire de l'unique réalité de la nature multiforme d'un texte vivant.

$$
\begin{aligned}
& \text { Liste des sigles } \\
& \begin{array}{ll}
\mathrm{LXX} \quad=\text { La Septante } \\
\mathrm{OG} \quad=\text { Old Greek } \\
\text { PentSam }=\text { Pentateuque Samaritain } \\
\mathrm{TM} \quad=\text { Texte Massorétique }
\end{array}
\end{aligned}
$$




\section{Références}

ALBRIGHT, W.F. New Light on Early Recensions of the Hebrew Bible. Bulletin of the American Schools of Oriental Research, Boston, Massachusetts, 140, p. 27-33, 1955.

AUSLOOS, H; LEMMELIJN, B. Content Related Criteria in Characterising the LXX Translation Technique. In: KRAUS, W.; KARRER, M.; MEISER, M. (Eds). Die Septuaginta - Texte, Theologien, Einflüsse. Tübingen: Mohr Siebeck, 2010. p. 357-376. (Wissenschaftliche Untersuchungen zum Neuen Testament, 252).

BOGAERT, P.-M. De Baruch à Jérémie. Les deux rédactions conservées du livre de Jérémie. In: BOGAERT, P.-M. (Ed.). Le livre de Jérémie. Leuven: Peeters, 1981. p. 168-173. (Biblioteca Ephemeridum Theologicarum Lovaniensium, 54).

BOGAERT, P.-M. Le livre de Jérémie en perspective. Les deux rédactions antiques selon les travaux en cours. Revue Biblique, Paris, v. 101, p. 363-406, 1994.

BOGAERT, P.-M. La Vetus Latina de Jérémie: texte très court, témoin de la plus ancienne Septante et d'une forme plus ancienne de l'hébreu (Jer 39 et 52). In: SCHENKER, A. (Ed.). The Earliest Text of the Hebrew Bible: the Relationship between the Masoretic Text and the Hebrew Base of the Septuagint Reconsidered. Atlanta, GA: Society of Biblical Literature, 2003. p. 51-82. (Septuagint and Cognate Studies Series, 52).

BROOKE, G. The Qumran Scrolls and the Demise of the Distinction Between Higher and Lower Criticism. In: CAMPBELL, J. G.; LYONS, W. J.; PIETERSEN, L. K. (Eds.). New Directions in Qumran Studies: Proceedings of the Bristol Colloquium on the Dead Sea Scrolls, 8-10 September 2003. London: T\&T Clark, 2005. p. 26-42. (Library of Second Temple Studies, 52).

CROSS, F. M. The Ancient Library of Qumran and Modern Biblical Studies. $3^{\text {rd }}$ ed. Sheffield: Sheffield Academic Press, 1995.

CROSS, F. M. From Epic to Canon: History and Literature in Ancient Israel: Baltimore/ London: John Hopkins University Press, 1998.

DAVILA, J. R. Text-type and Terminology: Genesis and Exodus as Test Cases. Revue de Qumrân, Paris, n. 16, p. 3-37, 1993.

DEBEL, H. Greek Variant Literary Editions to the Hebrew Bible? Journal for the Study of Judaism, Leiden, v. 41, p. 161-190, 2010.

DEBEL, H.; LEMMELIJN, B. De Rollen van de Dode Zee en de tekst (en) van het Oude Testament. In: DEBEL, H. (Ed.). De rollen van de Dode Zee. Vliebergh-Sencieleergang Sectie Bijbel 2010. Leuven: Acco/VBS, 2011. p. 163-210.

DEBEL, H. The Pluriformity of Pluriformity: a Reassessment of the Hermeneutical Framework for the Text-Critical Analysis of the Hebrew Bible. PhD Dissertation KU Leuven, 2011a.

DEBEL, H. Rewritten Bible, Variant Literary Editions and Original Text(s): Exploring the Implications of a Pluriform Outlook on the Scriptural Tradition. In: VON WEISSENBERG, H; MARTILLA, M.; PAKKALA, J. (Eds.). Changes in Scripture: Rewriting and Interpreting Authoritative Traditions in the Second Temple Period. 
Beihefte zur Zeitschrift für die Alttestamentliche Wissenschaft 419. Berlin/New York: De Gruyter, 2011b. p. 265-91.

DEBEL, H. Eeuwenoude teksten in een nieuw licht. De Dode Zeerollen, Qumran en de Bijbel, Antwerpen/Apeldoorn: Garant, 2012. p. 205-213.

DE LAGARDE, P. Anmerkungen zur griechischen Übersetzung der Proverbien, Leipzig, Brockhaus. 1863.

FISCHER, G. Die Diskussion um den Jeremiatext. In: KARRER, M.; KRAUS, W.; MEISER, M. (Eds.). Die Septuaginta Texte, Kontexte, Lebenswelten. Tübingen: Mohr Siebeck, 2008. p. 612-629. (Wissenschaftliche Untersuchungen zum Neuen Testament, 219).

HENDEL, R. Qumran and a New Edition of the Hebrew Bible. In: CHARLESWORTH, J.H. (Ed.). The Hebrew Bible and Qumran: the Bible and the Dead Sea Scrolls 1. Richland Hills, TX: Bibal, 2000a. p. 197-217.

HENDEL, R. The Text of the Torah after Qumran: Prospects and Retrospects. In: SCHIFFMAN, L.H.; TOV, E.; VANDERKAM, J.C. (Eds.). The Dead Sea Scrolls: Fifty Years after Their Discovery. Proceedings of the Jerusalem Congress, July 20-25, 1997. Jerusalem: The Israel Exploration Society, 2000b. p. 8-11.

HENDEL, R. Plural Texts and Literary Criticism: For Instance, 1 Samuel 17. Textus, n. 23, p. 97-114, 2007.

KAHLE, P. Untersuchungen zur Geschichte des Pentateuchtextes. Theologische Studien und Kritiken, Leipzig, v. 88, p. 399-439, 1915.

KÖNIG, E. Einleitung in das Alte Testament. Bonn: Weber, 1893.

KNEUCKER, J. J. F. Hitzig's Vorlesungen über biblische Theologie des Alten Testaments. Karlsruhe, 1880.

LEBRAM, H. C. M. Eine Streit um die Hebräische Bibel und die Septuaginta. In: SCEURLEER, T. H. L.; MEYJES, G. H. M. (Eds.). Leiden University in the Seventeenth Century. Leiden: Brill, 1975. p. 21-63.

LEMMELIJN, B. What are we Looking for in Doing Text-Critical Research? Journal of Northwest Semitic Languages, Leiden, v. 23, n. 2, p. 69-80, 1997.

LEMMELIJN, B. The So-Called "Major Expansions" in SamP, 4QpaleoExod ${ }^{\mathrm{m}}$ and 4QExod' Exod 7:14-11:10. On the Edge between Textual Criticism and Literary Criticism. In: TAYLOR, B. (Ed.). X Congress of the International Organization for Septuagint and Cognate Studies - Oslo 1998. Atlanta, GA: Society of Biblical Literature, 2001. p. 429-439. (Society of Biblical Literature. Septuagint and Cognate Studies Series, 51).

LEMMELIJN, B. A Plague of Texts?A Text-Critical Study of the So-Called 'Plagues Narrative' in Exodus 7,14-11,10. Leiden/Boston: Brill, 2009. (Oudtestamentische Studiën/Old Testament Studies, 56).

LEMMELIJN, B. Influence of a So-Called P-redaction in the 'Major Expansions' of Exod 7-11? Finding Oneself at the Crossroads of Textual and Literary Criticism. In: OTERO, A. P.; MORALES, P. T. (Eds.). Textual Criticism and Dead Sea Scrolls Studies in Honour of Julio Trebolle Barrera: Florilegium Complutense. 
Leiden/ Boston: Brill, 2012. p. 203-222. (Supplements to the Journal for the Study of Judaism, 157).

LEMMELIJN, B. Textual Criticism. In: SAlvesen, A.; Law. M. (Eds.). Oxford Handbook of the Septuagint. Oxford: Oxford University Press, 2020.

MILIK, J.T. Dix ans de découvertes dans le Désert de Juda. Paris: Cerf, 1957.

SANDERS, J.A. Canon and Community: a Guide to Canonical Criticism. Guides to Biblical Scholarship. Philadelphia, PA: Fortress, 1984. (Old Testament Series).

SANDERS, J.A. Stability and Fluidity in Text and Canon. In: NORTON, G.J.; PISANO, S. (Eds.). Tradition of the Text. FS D. Barthélemy. Freiburg: Universitätsverlag; Göttingen: Vandenhoeck \& Ruprecht, 1991. p. 203-217. (Orbis biblicus et orientalis , 109).

SCHENKER, A. What Do Scribes, and What Do Editors Do? The Hebrew Text of the Masoretes, the Old Greek Bible and the Alexandrian Philogical Ekdoseis of the $4^{\text {th }}$ and $3^{\text {rd }}$ Centuries B.C., Illustrated by the Example of 2Kings 1. In: AUSLOOS, H.; LEMMELIJN, B.; TREBOLLE BARRERA. J. (Eds.). After Qumran. Old and Modern Editions of the Biblical Texts: the Historical Books. Leuven/Paris/Walhole, MA: Peeters. 2012. p. 275-293. (Bibliotheca Ephemeridum Theologicarum Lovaniensium, 246).

SKEHAN, P. W. The Biblical Scrolls from Qumran and the Text of the Old Testament. The Biblical Archaeologist, v. 28, p. 87-100, 1965. [= CROSS, F.M.; TALMON, S. (Eds.). Qumran and the History of the Biblical Text. Cambridge. MA / London: Harvard University Press, 1975. p. 264-277].

STIPP, H.-J. Das Masoretische und alexandrinische Sondergut des Jeremiabuches, Textgeschichtlicher Rang, Eigenarten, Triebkräfte. Freiburg/Göttingen: University Press/ Vandenhoeck \& Ruprecht, 1994. (Orbis Biblicus et Orientalis, 136).

STIPP, H.-J. Linguistic Peculiarities in the Masoretic Edition of the Book of Jeremiah: An Updated Index. Journal of Northwest Semitic Languages, Leiden, v. 23, p. 181-202, 1997.

STIPP, H.-J. Zur aktuellen diskussion um das Verhältnis der Textformen des Jeremiabuches. In: KARRER, M.; KRAUS, W.; MEISER, M. Die Septuaginta Texte, Kontexte, Lebenswelten. Tübingen: Mohr Siebeck, 2008. p. 630-653. (Wissenschaftliche Untersuchungen zum Neuen Testament, 219).

STULMAN, L. Some Theological and Lexical Differences Between the Old Greek and the MT of the Jeremiah Prose Discourse. Hebrew Studies, New Berlin, n. 25, p. 18-23, 1984.

STULMAN, L. The Other Text of Jeremiah. A Reconstruction of the Hebrew text Underlying the Greek Version of the Prose Sections of Jeremiah with English Translation. Lanham, MD/ London: University Press of America, 1985.

TALMON, S. The Old Testament Text. In: ACKROYD, P. R.; EVANS, C. F. (Eds.). The Cambridge History of the Bible: from the Beginnings to Jerome. Cambridge: University Press, 1970. v. 1, p. 159-199.

TOV, E. A Modern Textual Outlook Based on the Qumran Scrolls. Hebrew Union College Annual, Cincinnati, n. 53, p. 11-27, 1982. 
TOV, E. The Text-Critical Use of the Septuagint in Biblical Research. Jerusalem, Simor, ${ }^{2}$ 1997. (Jerusalem Biblical Studies, 8).

TOV, E. The Many Forms of Hebrew Scripture: Reflections in Light of the LXX and 4QReworked Pentateuch. In: LANGE, A.; WEIGOLD, M.; ZSENGELLÉR, J. (Eds.). From Qumran to Aleppo: a Discussion with Emanuel Tov about the Textual History of Jewish Scriptures in Honor of his 65th Birthday. Göttingen: Vandenhoeck \& Ruprecht, 2009. p. 11-28. (Forschungen zur Religion und Literatur des Alten und Neuen Testaments, 230)

TOV, E. Textual Criticism of the Hebrew Bible. Third Edition. Revised \& Expanded. Minneapolis: Fortress Press. 2012a.

TOV, E. Post-Modern Textual Criticism?. In: LAW, T. M.; SALVESEN, A. (Eds.). Greek Scripture and the Rabbis. Leuven: Peeters, 2012b. p. 1-18. (Contributions to Biblical Exegesis and Theology, 66).

TREBOLLE BARRERA, J. The Jewish Bible and the Christian Bible: An Introduction to the History of the Bible. Leiden /New York/ Cologne: Brill, 1998.

TREBOLLE BARRERA, J. The Text-Critical Value of the Old Latin and Antiochean Greek Texts in the Books of Judges and Joshua. In: MARTINEZ, F. G.; VERVENNE, M. (Eds.). Interpreting Translation. Studies on the LXX and Ezekiel in Honour of Johan Lust. Leuven: University Press Peeters, 2005. p. 401-413. (Biblioteca Ephemeridum Theologicarum Lovaniensium, 192).

TREBOLLE BARRERA, J. A Combined Textual and Literary Criticism Analysis. Editorial Traces in Joshua and Judges. In: AUSLOSS, H.; LEMMELIJN, B.; VERVENNE, M. (Eds.). Florilegium Lovaniense. Studies in Septuagint and Textual Criticism in Honour of Florentino García Martínez. Leuven/Paris/Dudley, MA: Peeters, 2008. p. 437-463. (Bibliotheca Ephemeridum Theologicarum Lovaniensium, 224).

ULRICH, E. The Bible in the Making: the Scriptures at Qumran. In: ULRICH, E. U.; VANDERKAN, J.C. (Eds.). The Community of the Renewed Covenant: the Notre Dame Symposium on the Dead Sea Scrolls. Notre Dame: University of Notre Dame Press, 1994a. p. 77-93. (Christianity and Judaism in Antiquity, 10).

ULRICH, E. Multiple Literary Editions: Reflections toward a Theory of the History of the Biblical Text. In: PARRY, D.W; RICKS, S. D. (Eds.). Current Research and Technological Developments on the Dead Sea Scrolls. Conference on the Texts from the Judean Desert, Jerusalem, 30 April 1995. Leiden/New York/Cologne: Brill, 1994b. p. 78-105. (Studies on the Texts of the Desert of Judah, 20).

ULRICH, E. The Community of Israel and the Composition of the Scriptures. In: EVANS, C.A.; TALMON, S. (Eds.). The Quest for Context and Meaning: Studies in Biblical Intertextuality in Honor of James A. Standers. Leiden /New York /Cologne: Brill, 1997. p. 327-342. (Biblical Interpretation Series, 28).

ULRICH, E. The Dead Sea Scrolls and the Origins of the Bible. Grand Rapids, MI: Eerdmans, 1999.

ULRICH, E. The Dead Sea Scrolls and the Hebrew Scriptural Texts. In: CHARLESWORTH, J.H. (Ed.). The Hebrew Bible and Qumran: the Bible and the Dead Sea Scrolls 1. Richland Hills, Texas: Bibal, 2000. p. 105-133. 
WHITE CRAWFORD, S. Scribal Traditions in the Pentateuch and the History of the Early Second Temple Period. In: NISSINEN, M. (Ed.). Congress Volume Helsinki 2010. Leiden/Boston: Brill, 2012a. p. 167-184. (Supplements to Vetus Testamentum, 148).

WHITE CRAWFORD, S. Understanding the Textual History of the Hebrew Bible: a New Proposal. In: DAVID, N. et al. (Eds.). The Hebrew Bible in Light of the Dead Sea Scrolls. Göttingen: Vandenhoeck \& Ruprecht, 2012b. p. 60-69. (Forschungen zur Religion und Literatur des Alten und Neuen Testaments, 239).

Article soumis à l'evaluation le 10.01.2020 et approuvé le 30.03.2020.

Bénédicte Lemmelijn est Docteur en Théologie par la Katholieke Universiteit (KU) Leuven. Elle enseigne l'Ancien Testament au Département de Recherche d'Études Bibliques de la Faculté de Théologie et d'Études Religieuses de la KU Leuven, où elle dirige le Centre Louvain d'Études de la Septante et de Critique Textuelle. Elle est également associée de recherche à la Faculté de Théologie et de Religion de l'University of Free State (Afrique du Sud). Orcid .org/0000-0003-3458-3753. E-mail: benedicte.lemmelijn@kuleuven.be

Adresse: Tiensestraat 46

BE - 3440 Zoutleeuw - Belgium 